\title{
La probabilidad \\ conjunta en un proceso \\ de autovalidación
}

\author{
Fernando León Parada
}

\section{Resumen}

Este artículo contiene una parte de un informe de investigación sobre los procesos de autovalidación que desarrollaron estudiantes de ingeniería a través de un ambiente virtual de aprendizaje. Trata el análisis de los argumentos que encontraron los estudiantes de ingeniería en esquemas tipo mapa conceptual para tres situaciones problemáticas que, en pruebas de tres instancias distintas, les plantearon preguntas cerradas sobre probabilidad con opciones de respuesta que pretendían incurrirles en sesgos de razonamiento. Las tres situaciones de las instancias pretendían incurrir a los estudiantes en la falacia de la conjunción, alterando la regla de extensión y su independencia del contexto, y la segunda ley de De Morgan, debido a opciones ambiguas en el contexto. Las pruebas consistían en hacer que con los aprendizajes previos sobre el tema y la estrategia del mapa desarrollaran sus procesos de autovalidación de las respuestas que hubieran seleccionado al comienzo. Los resultados de las instancias coincidieron en que quienes desistían de sus respuestas inapropiadas fueron mayoría respecto de quienes insistían en las respuestas correctas. Este fenómeno didáctico hace parte de una disertación doctoral en el Doctorado Interinstitucional en Educación de la Universidad Distrital Francisco José de Caldas de Bogotá, Colombia.

Palabras clave: Ambientes virtuales de aprendizaje, autovalidación, falacia, probabilidad conjunta. 


\title{
The joint probability in a self-validation process
}

\begin{abstract}
This article contains a part of a research report on the self-validation processes that engineering students developed through a virtual learning environment. It deals with the analysis of the arguments that the engineering students found in conceptual map-type schemes for three problematic situations that, in tests of three different instances, asked them closed questions about probability with response options that were intended to incur them in reasoning biases. The three situations of the instances were intended to incur students in the fallacy of the conjunction, altering the extension rule and its independence from the context, and De Morgan's second law, due to ambiguous options in the context. The tests consisted in having the previous learning about the topic and the strategy of the map develops their self-validation processes of the answers they had selected at the beginning. The results of the instances agreed that those who gave up their inappropriate responses were the majority with respect to those who insisted on the correct answers. This didactic phenomenon is part of a doctoral dissertation at the Doctorado Interinstitucional en Educacion of the Universidad Distrital Francisco José de Caldas of Bogotá, Colombia.
\end{abstract}

Keywords: Fallacies, probability, self-validation, virtual learning environments.

Nombre del trabajo: La probabilidad conjunta en un proceso de autovalidación Nombre y apellido del autor: Fernando León Parada

Dirección completa: Calle 160 № 72-51, T2, A102, Bogotá, D.C., Colombia Lugar de trabajo: Universidad Distrital Francisco José de Caldas (UDFJC), Carrera 7 № 43-53, Piso 5, Of. Ingeniería de Sistemas, Ed. Admtvo. Bogotá, D. C., Colombia

Teléfono de contacto: Fijos: 571-4760617; 571-3239300, Ext: 2509. Celular: 304-4900-479 Dirección electrónica: profeleonp@gmail.com; (profeleonud@gmail.com) 


\section{Introducción}

El proceso de autovalidación de respuestas a preguntas de probabilidad es el objeto de investigación de una tesis doctoral titulada «El proceso de autovalidación de respuestas a preguntas capciosas sobre probabilidad en un ambiente virtual; estudio exploratorio con estudiantes de ingeniería», inscrita en el programa de Doctorado Interinstitucional en Educación (DIE-UD) de la Universidad Distrital Francisco José de Caldas (UDFJC) de Bogotá, Colombia. Este trabajo se centra en analizar los argumentos que encontraron los estudiantes de ingeniería en esquemas tipo mapa conceptual para tres situaciones problemáticas que, a través de un entorno virtual en pruebas de tres instancias distintas, les plantearon preguntas cerradas sobre la probabilidad con opciones de respuesta que pretendían incurrirles en sesgos de razonamiento. La intención de estos problemas fue la de hacer que seleccionaran respuestas inapropiadas para que utilizaran la estrategia del mapa, y con el cual podían encontrar argumentos que les resolviera el dilema de reafirmar la respuesta seleccionada 0 , por el contrario, retractarse de ella. Las dos primeras situaciones son ejemplos que se basan en la falacia de la probabilidad conjunta investigada por Tversky y Kahneman (1983), revisada por Díaz (2005) en un estudio exploratorio sobre «su posible solapamiento con el sesgo de equiprobabilidad.» (p. 50), y mencionada por Batanero, Contreras y Díaz (2012) como un «error (...) o creencia de que es más probable la intersección de dos sucesos que la de uno de ellos por separado o la de su unión» (p. 9). La tercera situación es una aportación propia que plantea una ambigüedad de tres opciones, siendo la tercera una equiprobabilidad basada en la equivalencia lógica de la negación de disyunciones y la conjunción de negaciones, conocida como segunda ley de De Morgan.

Las tres situaciones, aplicadas en distintos momentos con diferentes estudiantes de ingeniería, se caracterizaron por una significativa coincidencia: fueron mayoría quienes seleccionaron respuestas inapropiadas y después, con la ayuda de los esque- mas tipo mapa conceptuales, se retractaron de ellas, respecto de quienes insistieron en las respuestas correctas de cada prueba.

Este artículo contiene tres partes. La primera aclara algunos términos técnicos utilizados en la investigación tales como validación y autovalidación, estrategia del mapa conceptual (M), juicios finales de autovalidación, Ambiente Virtual de Aprendizaje Autorregulado (AVAA), Trayectoria Factual de Aprendizaje (TFA), y Falacia. En la segunda parte, se plantea el problema central de este aparte investigativo, el objetivo de su solución, una hipótesis, y algunas referencias de su marco teórico que inscriben este fenómeno como un problema de la disciplina de la didáctica de las matemáticas. La metodología aplicada siguió un plan de exploración in situ en tres instancias acontecidas en distintos momentos y diferentes estudiantes de ingeniería de la UDFJC. Al final, se redactan algunas conclusiones respecto de los procesos autónomos de autovalidación y del análisis de los resultados explorados, así como algunas recomendaciones para ampliar esta línea de investigación.

\section{Significado de términos de la investigación}

El término validación fue introducido por Brousseau (1987/2007) para distinguir una situación didáctica por la cual se institucionalizan los saberes en el aula de clase con una perspectiva desde la heterovalidación; afirmaba que el alumno requiere de un compañero de aula, a quien debe convencer de la validez de las afirmaciones que se hacen de las ideas para resolver un problema (p. 23).

Hubo otras posturas desde la perspectiva de la co-validación. Margolinas (1993/2009) considera este término para definir la fase de validación en la que el alumno decide él mismo sobre la validez de su trabajo, en un medio organizado bajo la responsabilidad del profesor en una situación a- 
didáctica (p.35); por su parte, Balacheff (2000) explica su proceso de validación como una actividad intelectual del estudiante que manipula información adquirida para producir una nueva información, cuando tiene como fin asegurarse de la validez de una proposición (p. 13).

Desde una perspectiva que enfatiza la facultad de la autonomía del estudiante, el término autovalidación en este trabajo se refiere al proceso mediante el cual un individuo utiliza una estrategia sin la participación sincrónica de tutores, para encontrar razones que le permitan determinar la validez o la invalidez de una respuesta a una pregunta.

El mapa conceptual es un término que define un recurso esquemático para representar un conjunto de significados conceptuales incluidos en una estructura de proposiciones (Novak y Gowin, 1984/1999: 33). El esquema tipo mapa conceptual de esta investigación se utiliza como estrategia didáctica para que el estudiante encuentre relaciones entre las aplicaciones de conceptos implicados por una pregunta y, a partir de su propio análisis, deduzca si una respuesta seleccionada anteriormente corresponde a una verdad coherente con los conceptos, o si es contradictoria.

Al final de la red de las aplicaciones de conceptos conectados en el Mapa se incluye una bifurcación con dos nodos flotantes, en uno aparece una frase que manifiesta insistir en la respuesta inicial, y en el otro, una frase que dice desistir de esa respuesta; cada una de estas frases es un juicio final de autovalidación, y aunque son mutuamente excluyentes, cuando un estudiante las señalaba juntas se interpretó que la última era una especie de autocorrección, elemento clave de la autorregulación, la que establece cualquier cambio autónomo en los criterios de interpretación, análisis, y tipos de decisión en un proceso; la aplicación web interactiva de mapas dotados con esta bifurcación se denominó Ambiente Virtual de Aprendizaje Autorregulado (AVAA). Simon (1995) introdujo el término Trayectoria Hipotética de Aprendizaje (THA) para indicar el posible camino del aprendizaje del estudiante en el aula, y advertía que era hipotética porque la trayectoria factual de aprendizaje no se puede conocer de antemano (p. 135); esta última expresión, Trayectoria Factual de Aprendizaje (TFA), se adoptó en este trabajo para indicar cualquiera de los caminos idiosincráticos de aprendizaje del estudiante que ocurrieran con el AVAA.

El concepto de Falacia mencionado en este trabajo corresponde a la definición: «Una falacia es un argumento no razonable o racionalmente no convincente, es decir que, aunque puede ser válido, contiene un error inferencial por violar uno o más criterios de buena argumentación» (Bordes, 2011:137).

\section{El problema de esta investigación}

El problema de este aparte investigativo se enfoca en la pregunta ¿qué razones justifican que un mapa conceptual incida en el proceso de autovalidación de una respuesta dada por el estudiante a una pregunta vinculada con la probabilidad conjunta? El objetivo es analizar los elementos de los esquemas tipo mapa conceptual que le dan razones a un estudiante para que lleve a cabo el proceso de autovalidación de una respuesta seleccionada de tres opciones posibles, para una pregunta sobre probabilidad conjunta.

La hipótesis de trabajo se formuló así: El uso del mapa conceptual promueve el aprendizaje en el estudiante, para que incorpore la filtración de soluciones tentativas a un problema donde interviene la probabilidad conjunta, y para que desarrolle su proceso de autovalidación de una solución definitiva. Las unidades de análisis fueron las acciones autónomas registradas por un subsistema del AVAA de las TFA de los estudiantes; cada TFA fue expresada como una cadena o concatenación de los códigos que identificaban las interacciones de consulta en el mapa y determinaciones en los nodos de juicio final de autovalidación. 


\section{Marco Teórico}

El marco teórico del problema de investigación se relaciona con las falacias de la probabilidad conjunta y de la probabilidad disyunta. Díaz (2005: 45) en un artículo sobre la evaluación de la falacia de la conjunción en alumnos universitarios señala que

«Las personas, en su vida cotidiana, (...). En lugar de usar un cálculo de probabilidades normativo, confian en reglas relativamente simples llamadas heurísticas que son las que guían sus juicios».

Además la regla de extensión en la teoría de probabilidad establece lo siguiente: si la extensión de A incluye la extensión de $B$ (es decir, A contiene a $B$ ), entonces $P(A) \geq P(B)$. Dado que el conjunto de las posibilidades asociado a la conjunción $A \& B$ está incluido en el conjunto de las posibilidades asociadas con $B$, el mismo principio puede expresarse para la conjunción $\mathrm{P}(\mathrm{A} \& \mathrm{~B}) \leq \mathrm{P}(\mathrm{B})$ (Tversky y Kahneman, 1983: 294). Un caso especial de esta regla es la que se aplica a la conjunción, la cual establece que la probabilidad de $A \& B$ no puede exceder a la probabilidad de $A$ ni a la probabilidad de B, por ser subconjunto de cada uno de ellos.

Tversky y Kahneman (1983/2002) demostraron que, bajo ciertas circunstancias, las personas violan de manera predecible y sistemática esta regla de la conjunción; en varios casos comprobaron que señalaban mayor probabilidad a la conjunción de los eventos que a uno de los eventos que la conforman. Respecto de la dualidad lógica versus intuición, concluyeron que el error inducido en la falacia de la conjunción demuestra claramente el contraste entre la lógica extensional, que subyace a la mayoría de los conceptos formales de la probabilidad, y las consideraciones naturales que rigen muchos juicios y creencias (p. 43).

Otro caso especial de la regla de extensión es la que se aplica a la disyunción, la cual establece que la probabilidad de A- O - B no puede ser menor que la probabilidad de $A$ ni que la probabilidad de B, porque les contiene a ambos. Bar-Hillel y Neter
(1986/2002) se motivaron en el estudio de cómo crear una falacia de disyunción teniendo en cuenta que, al categorizar las cosas por sus condiciones de certeza, las personas prefieren colocar las de nivel básico como categorías superiores. Si se exhibiera la misma preferencia en la categorización de condiciones de incertidumbre, entonces podría surgir la falacia de la disyunción. Esto no solo sería una extensión de la falacia de la conjunción, sino que también se basaría en un mecanismo cognitivo completamente diferente al evocado con la heurística de la representatividad (p. 96).

Dunning, Meyerowitz, y Holzberg (1989/2002) encontraron que las personas suelen seleccionar una definición cuando ella les sea favorable para un propósito. Muchos investigadores han argumentado que las personas otorgan gran importancia a la evidencia que es consistente con los resultados deseados, mientras que descartan fuertemente la evidencia contraria (p. 325).

Los textos de enseñanza son precisos en cuanto a sus definiciones. Por ejemplo, la probabilidad se define como una función cuyo dominio son los conjuntos que representan los eventos posibles de un experimento aleatorio; los criterios de existencia y unicidad de tal función de probabilidad permiten concluir que, si dos eventos están definidos por los conjuntos $A$ y $B$, respectivamente, y si $A$ es igual a $B$, entonces, los eventos $A$ y $B$ tienen igual probabilidad.

En la lógica formal, las leyes de De Morgan expresan que 1) la negación de la conjunción es equivalente a la disyunción de las negaciones, y 2) la negación de la disyunción es equivalente a la conjunción de las negaciones; las transferencias de estas reglas a la teoría de conjuntos establecen que: 1) el complemento de la intersección de conjuntos es igual a la unión de los complementos de esos conjuntos; 2) el complemento de la unión de conjuntos es igual a la intersección de los complementos de esos conjuntos. $\mathrm{Si}$ C y T son eventos indicamos con un apóstrofo a sus eventos complementarios $C^{\prime}$ y $T^{\prime}$; si $P[A]$ significa el valor de probabilidad de un evento $A$, las leyes 
de De Morgan aplicadas a la probabilidad con los eventos $C$ y $T$, formalmente son:

1) $\mathrm{P}\left[(\mathrm{C} \cap \mathrm{T})^{\prime}\right]=\mathrm{P}\left[\mathrm{C}^{\prime}\right] \cup \mathrm{P}\left[\mathrm{T}^{\prime}\right]$; 2) $\mathrm{P}\left[(\mathrm{C} \cup \mathrm{T})^{\prime}\right]=$ $\mathrm{P}\left[\mathrm{C}^{\prime}\right] \cap \mathrm{P}\left[\mathrm{T}^{\prime}\right]$

Los eventos que son equiprobables bajo las leyes de De Morgan no son claros para ciertas personas por asignarles valores distintos de probabilidad. Cuando se emplean rasgos muy amplios que causan ambigüedad en las personas, provocan en ellas el uso de definiciones idiosincráticas (Duning, et.al., 1989/2002).

\section{Metodología}

El desarrollo metodológico siguió un plan de tres instancias cada una con un grupo de (30) treinta estudiantes de pregrado en ingeniería que habían recibido las mismas clases presenciales sobre el tema de la probabilidad conjunta, de la asignatura de probabilidad impartida en la UDFJC. La agenda estrenó la Situación problema 1 en septiembre de 2016 con una prueba en el AVAA que debían contestar 30 estudiantes.

Luego, después de un refinamiento de la prueba se programó una nueva prueba con la Situación problema 2, que se realizó en octubre de 2017 con la participación de otro grupo de 30 estudiantes con la misma condición; la razón del tiempo extendido fue por aspectos logísticos y por garantizar que ninguno tenía conocimiento sobre la prueba. Posteriormente, se proyectó el estreno de la prueba con la Situación problema 3 para el mes de septiembre de 2018 en la que participaron otros 30 estudiantes con iguales condiciones de otro curso de ingeniería de la UDFJC. El método planeado cotejó los resultados cuantificables en términos de las frecuencias de quienes adoptarían cada una de las formas del proceso de autovalidación en una situación problema. El análisis de los resultados de las tres situaciones problema se hizo teniendo como base los contrastes y las coincidencias significativas de las regularidades y de casos atípicos.

\section{Resultados}

En esta sección se presentan los cuatro posibles casos de cada situación problema, según sus respectivas premisas y esquemas tipo mapa conceptual, que los estudiantes analizaron, de modo individual y autónomo, para determinar luego, si debían insistir en su respuesta seleccionada por considerarla apropiada, o en desistir de ella si le encontraba alguna contradicción. Los resultados de cada prueba indican términos de frecuencias absolutas de cuantos estudiantes efectuaron cada forma del proceso de autovalidación según las opciones de respuesta de cada situación problema, y siguen con el análisis respectivo y la conclusión pertinente.

Las situaciones de las pruebas son del caso de la conjunción, y en todas se aplica la regla de extensión: la probabilidad de un evento nunca es mayor que la probabilidad de cualquiera de sus extensiones, ni menor que la probabilidad de un evento que contenga propiamente. $(\mathrm{C} \cap \mathrm{T}) \subsetneq \mathrm{C}$ implica que $\mathrm{P}(\mathrm{C} \cap \mathrm{T})<\mathrm{P}(\mathrm{C})$. El evento de haber asistido a Clase se denota $C$, el evento de haber hecho la Tarea se denota T, el apóstrofo en la letra que denota un evento, o fuera de la expresión parentética de una operación conjuntista, indica un evento complementario.

\section{Situación problema 1.}

Un profesor no llamaba a lista para comprobar la asistencia a Clase de sus estudiantes, sino que, les enviaba una Tarea del tema visto para evaluarlo con una pregunta en un Quiz de la Clase siguiente; calificaba cada Quiz como «Aprobado» si la respuesta a la pregunta era correcta, o «Reprobado» si era incorrecta. Si un estudiante tenía en su Quiz la nota «Aprobado»; ¿Cuál evento, previo a la presentación de la prueba sería el más probable? Seleccione su opción de respuesta:

a) Asistió a Clase.

b) Asistió a Clase e hizo la Tarea.

c) Las anteriores opciones son igual de probables. Los siguientes esquemas tipo mapa conceptual contienen los conceptos relacionados con el tema de la pregunta de esta situación, los cuales fueron de ayuda para que los estudiantes analizaran la situación y llevaran a cabo los procesos de autovalidación. 
Primer caso. Premisa: Algunos, no todos, quienes fueron a Clase, hicieron la Tarea; y no todos, quienes hicieron la Tarea, fueron a Clase.

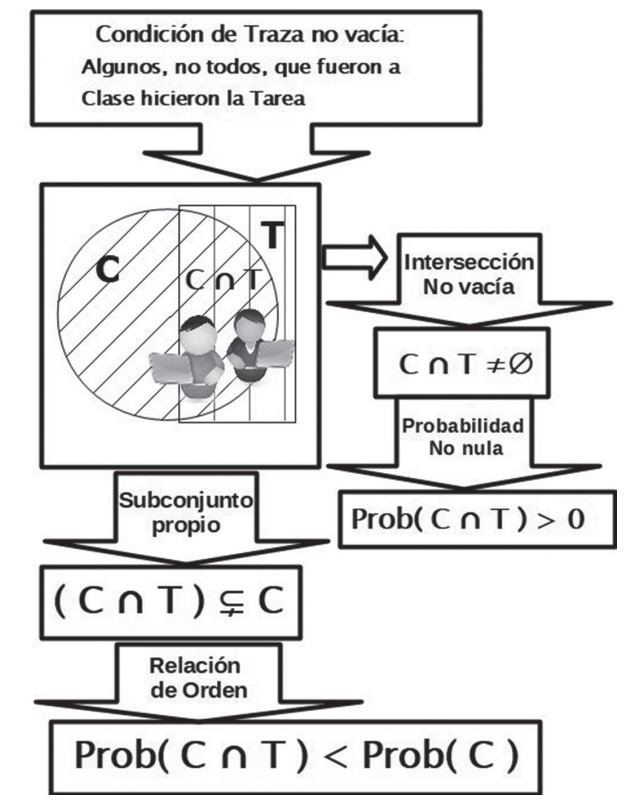

Tercer caso. Premisa: Todos, quienes fueron a Clase, hicieron la Tarea; y algunos, que hicieron la Tarea, no fueron a Clase.

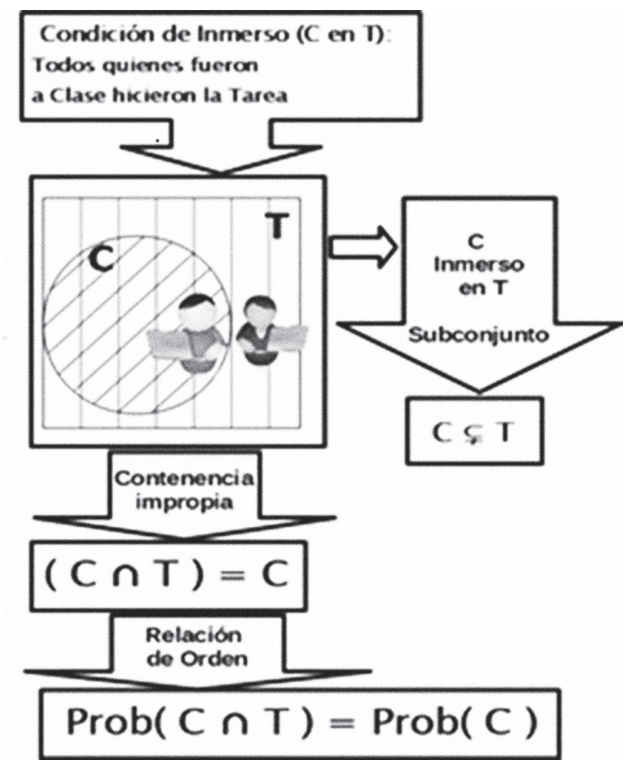

Segundo caso. Premisa: Todos, quienes fueron a Clase, no hicieron la Tarea; $y$ todos, quienes hicieron la Tarea, no fueron a Clase.

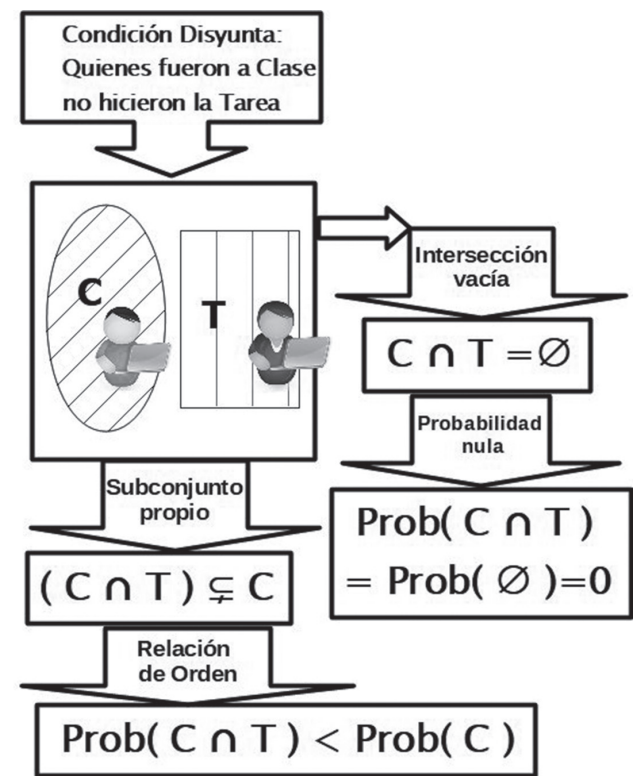

Cuarto caso. Premisa: Todos, quienes hicieron la Tarea, fueron a Clase; y algunos, que fueron a Clase, no hicieron la Tarea.

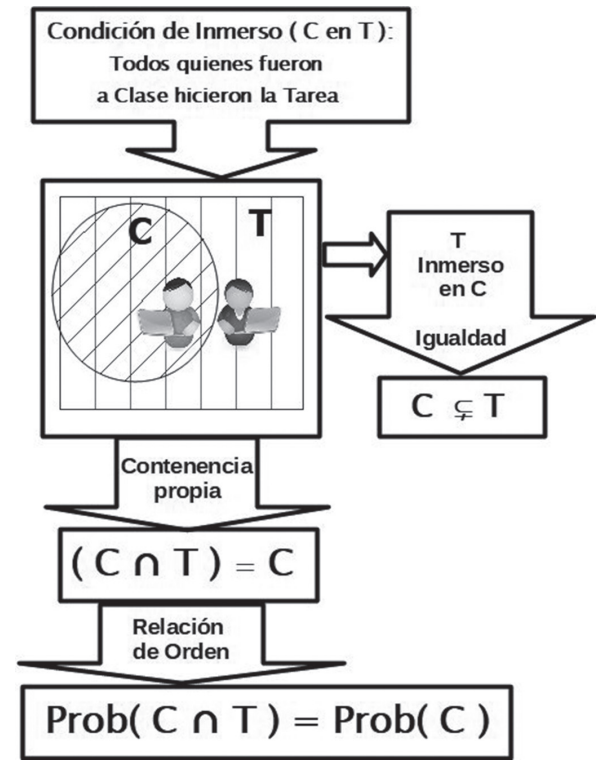

Cuadro 1.

Cuatro esquemas tipo mapa conceptual para la situación problema 1. (Fuente propia) 
La Tabla 1 tiene las frecuencias de los estudiantes en sus procesos de autovalidación.

\begin{tabular}{|l|c|c|c|c|}
\hline & $\begin{array}{c}\text { Responde } \\
\text { a) }\end{array}$ & $\begin{array}{c}\text { Responde } \\
\text { b) }\end{array}$ & $\begin{array}{c}\text { Responde } \\
\text { c) }\end{array}$ & Total \\
\hline Insisten & 2 & 1 & 3 & 6 \\
Desisten & 1 & 20 & 3 & 24 \\
Total & 3 & 21 & 6 & 30 \\
\hline
\end{tabular}

\section{Tabla 1.}

Resultados de los procesos de autovalidación de la prueba con la situación problema 1.

(Fuente propia)

Estos resultados evidenciaron presencia de sesgos de razonamiento; las frecuencias absolutas aumentadas en el tamaño de fuente, son 20 y 3 , la frecuencia relativa del $23 / 30=76.7 \%$ es alta e indica que la mayoría de los estudiantes desistieron de sus respuestas por encontrarlas inapropiadas después de haber encontrado argumentos en los cuatro esquemas tipo mapa conceptual.

La conclusión de esta prueba es la siguiente: El sesgo se presenta por creer que $C \&$ T es más influyente que $C$, es decir, pensar que la probabilidad de una conjunción fuese una suma de probabilidades como si fuera que «total es mayor que cualquiera de sus partes». Esto no es de ningún caso. Un sesgo especial se presenta con la respuesta c), al suponer que solo puede ocurrir el tercer caso: Todos, quienes fueron a Clase, hicieron la Tarea; y algunos, que hicieron la Tarea, no fueron a Clase.

\section{Situación problema 2.}

Un profesor no llamaba a lista para comprobar la asistencia a Clase de sus estudiantes, sino que, les enviaba una Tarea del tema visto para evaluarlo con una pregunta en un Quiz de la Clase siguiente; calificaba cada Quiz como «Aprobado» si la respuesta a la pregunta era correcta, 0 «Reprobado» si era incorrecta. Si un estudiante tenía en su Quiz la nota «Reprobado»; ¿Cuál evento, previo a la presentación de la prueba, sería el más probable? Seleccione su opción de respuesta:

a) No fue a Clase.

b) No fue a Clase ni hizo la Tarea.

c) De las anteriores opciones, no hay una que sea la más probable.

La opción a) es el evento $C^{\prime}$. La opción b) es el evento $C^{\prime} \cap T^{\prime}$. Los siguientes esquemas tipo mapa conceptual contienen los conceptos relacionados con el tema de la pregunta de esta situación problema 2, necesarios para que los estudiantes hicieran el análisis de los argumentos que utilizaron en los procesos de autovalidación. 
Primer caso. Premisa: Quienes no fueron a Clase ni hicieron la Tarea, no eran todos los que no fueron a Clase.
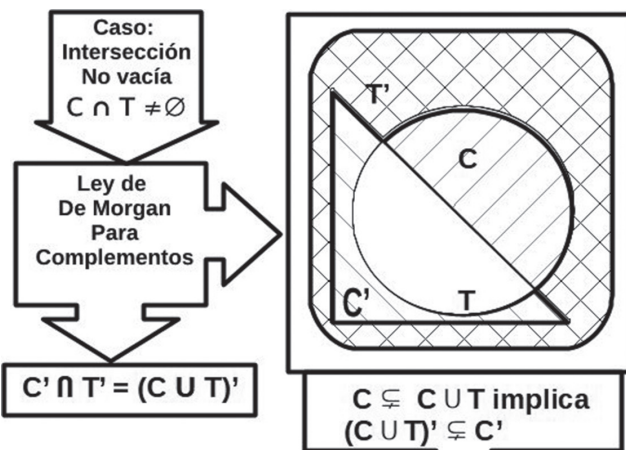

$(C \cup T)^{\prime} \subsetneq C^{\prime}$

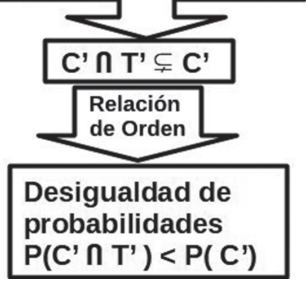

Tercer caso. Premisa: Todos, quienes fueron a Clase, hicieron la Tarea; pero algunos que hicieron la Tarea, no fueron a Clase.

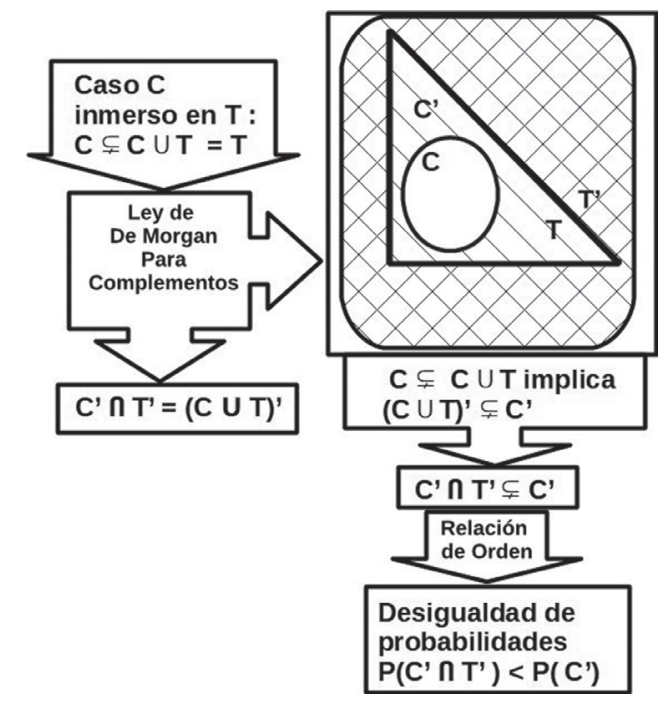

Segundo caso. Premisa: Todos, quienes fueron a Clase, no hicieron la Tarea; $y$ todos, quienes hicieron la Tarea, no fueron a Clase.

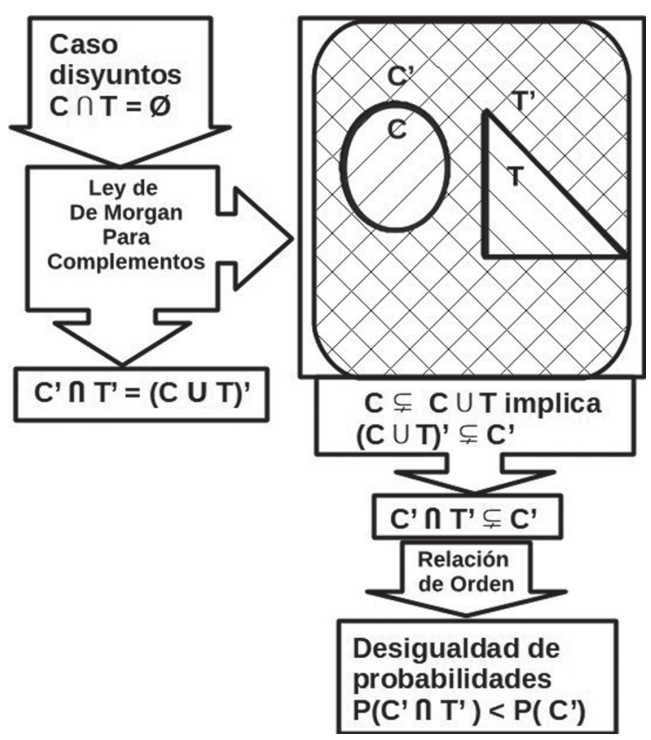

Cuarto caso. Premisa: Todos, quienes hicieron la Tarea, fueron a Clase; pero algunos que fueron a Clase, no hicieron la Tarea.

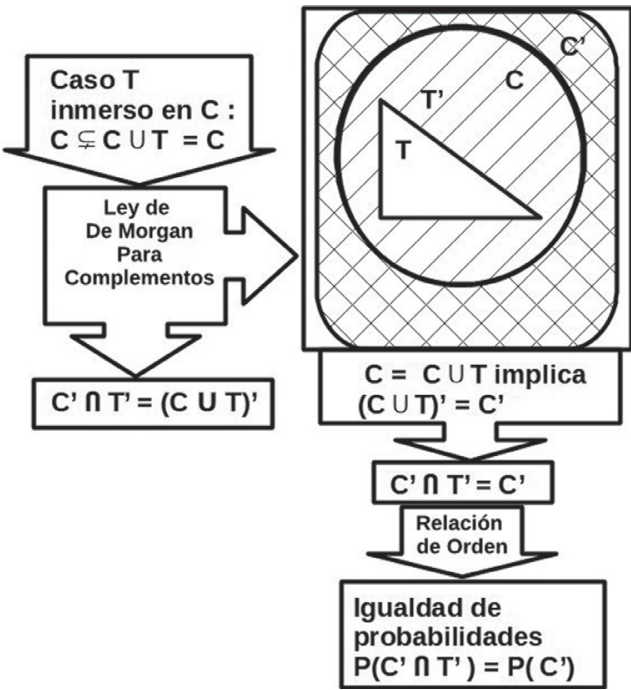

Cuadro 2.

Cuatro esquemas tipo mapa conceptual para la situación problema 2. (Fuente propia) 
La Tabla 2 tiene las frecuencias de los estudiantes en sus procesos de autovalidación.

\begin{tabular}{|l|c|c|c|c|}
\hline & $\begin{array}{c}\text { Responde } \\
\text { a) }\end{array}$ & $\begin{array}{c}\text { Responde } \\
\text { b) }\end{array}$ & $\begin{array}{c}\text { Responde } \\
\text { c) }\end{array}$ & Total \\
\hline Insisten & 1 & 2 & 2 & 5 \\
Desisten & 1 & 17 & 7 & 25 \\
Total & $\mathbf{2}$ & $\mathbf{1 9}$ & $\mathbf{9}$ & $\mathbf{3 0}$ \\
\hline
\end{tabular}

\section{Tabla 2.}

Resultados de los procesos de autovalidación de la prueba con la situación problema 2.

(Fuente propia)

La presencia del sesgo de razonamiento se evidencia en las frecuencias absolutas de las opciones b) y c) donde se ha aumentado el tamaño de la fuente, 17 y 7 , suman 24 y corresponden a la frecuencia relativa de valor $24 / 30=80 \%$, elevada proporción de estudiantes que desistieron de sus respuestas que contradecían las ideas relacionadas en los esquemas tipo mapa conceptual de los casos de esta situación problema 2 .

La conclusión de esta prueba es la siguiente: El sesgo se presenta por creer que $C^{\prime} \& T^{\prime}$ es más influyente que $C^{\prime}$, es decir, pensar que la probabilidad de una conjunción fuese la suma de las probabilidades de los eventos que la componen, en esta situación, independientemente que se trata de eventos complementarios de otros eventos, desatendieron el contexto de la regla de extensión:

$\left(C^{\prime} \cap T^{\prime}\right) \subsetneq C^{\prime}$ implica que $P\left(C^{\prime} \cap T^{\prime}\right)<P\left(C^{\prime}\right)$.

Un sesgo especial se presentó con la respuesta c), al suponer que solo puede ocurrir el cuarto caso: Todos, quienes hicieron la Tarea, fueron a Clase; pero algunos que fueron a Clase, no hicieron la Tarea.

\section{Situación problema 3.}

Un profesor no llamaba a lista para comprobar la asistencia a Clase de sus estudiantes, sino que, les enviaba una Tarea del tema visto para evaluarlo con una pregunta en un Quiz de la Clase siguiente; calificaba cada Quiz como «Aprobado» si la respuesta a la pregunta era correcta, 0 «Reprobado» si era incorrecta.

Si un estudiante tenía en su Quiz la nota «Reprobado»; ¿Cuál evento, previo a la presentación de la prueba, sería el más probable? Seleccione su opción de respuesta:

a) No estar entre los que fueron a Clase o hicieron la Tarea.

b) Estar entre los que no fueron a Clase ni hicieron la Tarea.

c) De las anteriores opciones, no hay una que sea la más probable.

La opción a) es el evento $(C \cup T)^{\prime}$. La opción b) es el evento $C^{\prime} \cap T^{\prime}$.

A continuación, se explican los posibles casos que el estudiante analizaba para determinar si su respuesta seleccionada era coherente o contradictoria. 
Primer caso. Premisa: Quienes no fueron a Clase $n i$ hicieron la Tarea, no eran todos los que no fueron a Clase.

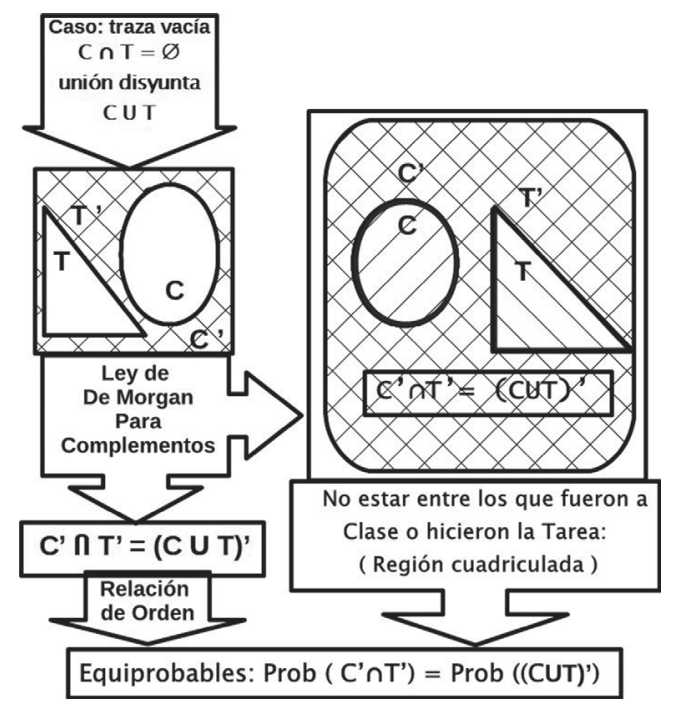

Tercer caso. Premisa: Todos, quienes fueron a Clase, hicieron la Tarea; pero algunos que hicieron la Tarea, no fueron a Clase.

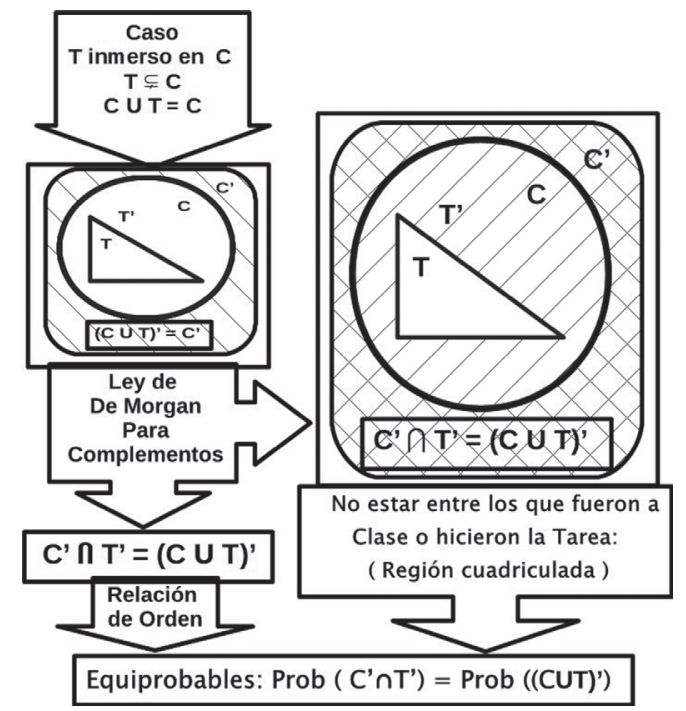

Segundo caso. Premisa: Todos, quienes fueron a Clase, no hicieron la Tarea; $y$ todos, quienes hicieron la Tarea, no fueron a Clase.

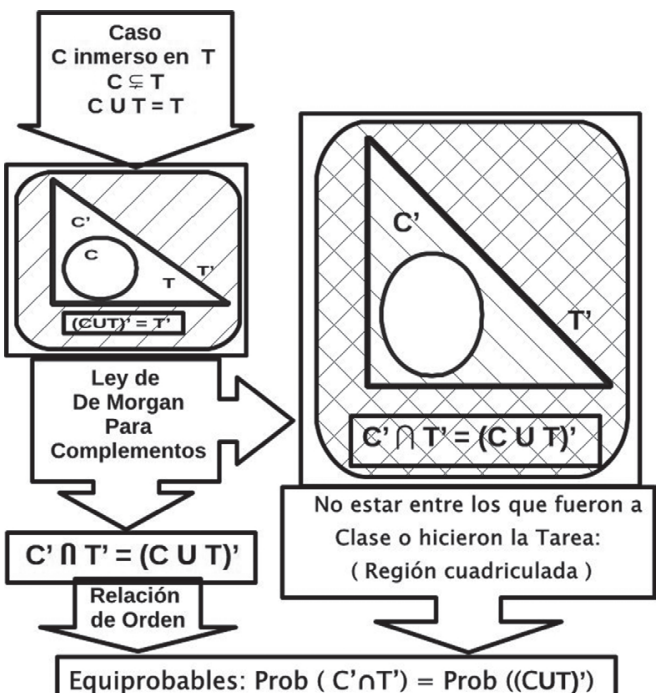

Equiprobables: Prob ( C'nT') = Prob ((CUT)')

Cuarto caso. Premisa: Todos, quienes hicieron la Tarea, fueron a Clase; pero algunos que fueron a Clase, no hicieron la Tarea.

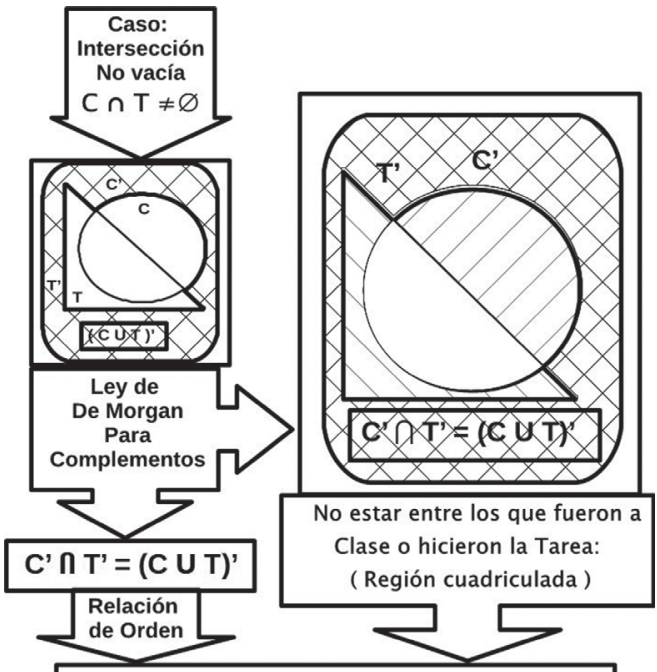

Equiprobables: Prob $\left(C^{\prime} \cap T^{\prime}\right)=$ Prob ((CUT)')

Cuadro 3.

Cuatro esquemas tipo mapa conceptual para la situación problema 3. (Fuente propia) 
La Tabla 3 tiene las frecuencias de los estudiantes en sus procesos de autovalidación.

\begin{tabular}{|l|c|c|c|c|}
\hline & $\begin{array}{c}\text { Responde } \\
\text { a) }\end{array}$ & $\begin{array}{c}\text { Responde } \\
\text { b) }\end{array}$ & $\begin{array}{c}\text { Responde } \\
\text { c) }\end{array}$ & Total \\
\hline Insisten & 2 & 3 & 3 & 8 \\
Desisten & 8 & 11 & 3 & 22 \\
Total & 10 & 14 & 6 & 30 \\
\hline
\end{tabular}

Tabla 3.

Resultados de los procesos de autovalidación de la prueba con la situación problema 3.

(Fuente propia)

La presencia del sesgo de razonamiento se evidencia en las frecuencias absolutas de las opciones $a$ ) y b) donde se ha aumentado el tamaño de la fuente, 8 y 11 , que suman 19 y corresponden a una significativa frecuencia relativa de valor $19 / 30=63.3 \%$, lo cual indica que fue mayoría en los estudiantes que desistieron de sus respuestas, al descubrir contradicciones de sus respuestas con las ideas relacionadas en los esquemas tipo mapa conceptual de los casos de esta situación problema 3.

La conclusión de esta prueba es la siguiente: El sesgo se presenta por desconocer la $2^{2}$. Ley de De Morgan, que establece $(C \cup T)^{\prime}=C^{\prime} \cap T^{\prime}$, y en consecuencia por los criterios de existencia y unicidad de la función de probabilidad:

$\mathrm{P}\left[(\mathrm{C} \cup \mathrm{T})^{\prime}\right]=\mathrm{P}\left[\mathrm{C}^{\prime}\right] \cap \mathrm{P}\left[\mathrm{T}^{\prime}\right]$

La causa de este sesgo de razonamiento indicada por el desconocimiento de la regla de equivalencia entre la negación de la disyunción y la conjunción de las negaciones, pudo no ser la única, es posible que el estudiante haya incurrido en la falacia de la disyunción con los eventos C y T, antes de considerar el complemento de su unión; y otra causa posible también pudo haberse dado por la ambigüedad de las opciones a) y b) que aparentaban ser diferentes cuando eran excluidas del contexto.

\section{Discusiones, conclusiones y recomendaciones}

7.1. Discusiones

Las pautas de discusión sobre los resultados se iniciaron con estas preguntas.

¿Es la falacia de la conjunción un fenómeno psicológico que afecta el aprendizaje de los principios básicos de la probabilidad?

¿Qué dificultades tiene la didáctica tradicional que es permeable frente a este fenómeno y permite incurrir en un sesgo tan particular a estudiantes de ingeniería, que aún tenían una aceptable formación en los fundamentos de la matemática y estudiados previamente conceptos básicos de probabilidad?

\subsection{Conclusiones}

1) El estudio de los procesos de autovalidación provee una alternativa para:

- Poner a prueba la potencia de la estrategia de los mapas conceptuales en el desarrollo de los procesos autónomos dirigidos a la formación en las ciencias básicas de ingeniería; en esta exploración, el estudiante descubre el error de su respuesta inapropiada cuando contrasta su condición en un diagrama de un mapa conceptual, lo cual está en la dirección de lo citado por Díaz (2005):

«Crandall y Greenfield (1986) enseñaron sistemáticamente a sus sujetos a usar diagramas de Venn para resolver problemas similares a los descritos anteriormente, encontrando una mejoría moderada en el grupo experimental de sujetos». (p. 48);

- Destacar evidencias de fenómenos ocultos en el pensamiento aleatorio; en este caso el estudiante es quien se obliga con la autovalidación de su respuesta, apoyado en el mapa como herramienta didáctica, para enfrentar un fenómeno psicológico.

2) Es útil conocer debilidades en el campo de la didáctica disciplinar; Díaz (2005: 48) cita: 
"... estudios en los que se fuerza a los alumnos a considerar sus respuestas y producen alguna mejora en la resolución de las tareas se describen en Sedlemeier (1999)...»

para mejorar la comprensión de las probabilidades compuestas.

La enseñanza virtual supera dificultades mantenidas por la didáctica tradicional que ha sido permeable a los efectos de fenómenos de perturbación y de deficiencia cognitiva inherentes al azar como ocurrió con las respuestas inapropiadas. El proceso autónomo del estudiante de ingeniería que reconoce haber seleccionado una respuesta inapropiada a una pregunta sobre la probabilidad conjunta, y después con el esquema tipo mapa conceptual logra hallar razones por las que debe retractarse de esa respuesta, sin la ayuda de tutores sincrónicos, evidencia el efecto de los procesos de autovalidación en ambientes virtuales de aprendizaje.

3) La hipótesis mencionada al inicio se cumplió a cabalidad en las tres exploraciones: El uso del mapa conceptual promueve el aprendizaje en el estudiante, para que incorpore la filtración de soluciones tentativas a un problema donde interviene la probabilidad conjunta, y para que desarrolle su proceso de autovalidación de una solución definitiva.

\subsection{Recomendaciones}

Esta experiencia investigativa puede ser fortalecida con experiencias de aula, investigaciones de docentes y aportes de investigadores y expertos; equivocaciones frecuentes de los estudiantes posiblemente ocultan casos de muchas falacias.

La investigación parcializada sobre la probabilidad conjunta es de fácil comprensión, pero se recomienda profundizar un poco más con otras pruebas, como las que ya se realizaron con el AVAA, donde las TFA evidenciaron resultados muy similares en situaciones problemáticas asociadas a las falacias: de la probabilidad disyunta, de la probabilidad condicional, de la probabilidad de eventos independientes, del valor esperado, de la presencia de sesgos muestrales.

La última recomendación es el manejo del concepto de autorregulación, el cual le permite al estudiante «reversar» una decisión equivocada sin el temor de una sanción generada externamente, el mismo proceso de autorregulación permite al estudiante modificar o ajustar sus propios criterios para la auto-corrección y organización de las decisiones, previos a la determinación de los juicios finales de autovalidación. 


\section{Referencias bibliográficas}

Bordes, S.M. (2011). Las trampas de Circe: falacias lógicas y argumentación informal. Madrid: Ediciones Cátedra.

Balacheff, N. (2000). Procesos de prueba en los alumnos de matemáticas. (Traducción: Pedro Gómez; Colaboración: Ángela Pinilla). Santafé de Bogotá: Universidad de los Andes (Colombia), Una Empresa Docente.

Brousseau, G. (2007). Iniciación al estudio de la teoría de las situaciones didácticas. (Dilma Fregona, trad.). Buenos Aires: Libros del Zorzal. (Obra original publicada en 1986).

Bar-Hillel, M. y Neter, E. (1993/2002). How Alike Is It? versus How Likely Is It?: A Disjunction Fallacy in Probability Judgments. En T. Gilovich, D. Griffin, y D. Kahneman (Eds.), Heuristics and Biases (pp. 82-97). New York, NY: Cambridge University Press. (Original work published 1993. Journal of Personality and Social Psychology, 65, 1119-1131).

Batanero, C., Contreras, J.M. y Díaz, C. (2013). Sesgos en el razonamiento sobre probabilidad condicional e implicaciones para la enseñanza. Revista Digital Matemática, 12(2), 1-13. Disponible en http://funes.uniandes.edu.co/8042/

Crandall, C.S., \& Greenfield, B. (1986). Understanding the conjunction fallacy: A conjunction of effects? Social Cognition, 4(4), 408-419.

Díaz, C. (2005). Evaluación de la falacia de la conjunción en alumnos universitaios. Suma: Revista sobre Enseñanza y Aprendizaje de las Matemáticas, (48), 45-50. Disponible en: https://dialnet. unirioja.es/servlet/articulo?codigo $=1122234$

Dunning, D., Meyerowitz, J.A. y Holzberg, A.D. (1989/2002). Ambiguity and self-evaluation: The role of idiosyncratic trait definitions in self-serving assessments of ability. En T. Gilovich, D. Griffin, y D. Kahneman (Eds.), Heuristics and Biases (pp. 324-333). New York, NY: Cambridge University Press. (Original work published 1989. Journal of Personality and Social Psychology, 57, 1082-1090). Margolinas, C. (2009). La importancia de lo verdadero y de lo falso en la clase de matemáticas (Martín E. Acosta G., y Jorge E. Fiallo L., trads.). Bucaramanga, Colombia: Universidad Industrial de Santander. (Obra original publicada en 1993).

Novak, J.D. y Gowin, D.B. (1999). Aprendiendo a aprender. (J.M. Campanario, E. Campanario, trads.). Barcelona: Martínez Roca. (Versión original publicada en 1984).

Sedlmeier, P. (1999). Improving statistical reasoning. Theoretical models and practical implications. Mahwah, NJ: Erlbaum.

Simon, M. A. (1995). Reconstructing mathematics pedagogy from a constructivist perspective. Journal for Research in Mathematics Education, 26(2), 114-145. doi:10.2307/749205

Tversky, A. y Kahneman, D. (1983). Extensional versus intuitive reasoning. The conjunction fallacy in probability judgment, Psychological Review, 90, 293-315. 